\title{
A SURVEY ON CONTEMPORARY TERRESTRIAL AND HANDHELD MULTIMEDIA BROADCASTING NETWORKS
}

Digital video broadcasting (DVB) transmission technologies are suitable for wireless delivery of multimedia content both to static and mobile end-users. On the one hand, DVB-T standard has been adopted for digital terrestrial television (DTT) and it has been further developed into a newer standard, DVB-T2, which was recently finalized. On the other hand, DVB-H is a specification for the transmission of digital $T V$ to handheld user terminals such as smart phones delivering IP-encapsulated data. The combination of satellite and terrestrial DVB technologies is DVB-SH which delivers wide coverage to mobile terminals and promises upgraded services to mobile and vehicle users via a satellite link. The objective of our research article is to present a survey on terrestrial, mobile and hybrid DVB multimedia networks. Emphasis will be given on quality of radio and service related aspects. A focus on performance evaluation methodology based mainly on measurement campaign will be proposed with a detailed presentation of experimental equipment for drive-test field measurements. Guidelines of measurements and analysis processes will be described. A public DVB network in the area of Athens will be our case study.

Keywords: DVB-T/T2, DVB-H/SH, Multimedia Broadcasting, Quality of Service, Performance Evaluation, Measurement Campaign.

\section{Introduction}

Digital video broadcasting (DVB) systems [1] are suitable for multimedia content delivery both to fixed and mobile end-users. In Europe, DVB-T (Terrestrial) standard [2] has been adopted for terrestrial digital wireless transmission. DVB-T has been further developed into newer standards such as DVB-H (Handheld) [3], now in operation, and DVB-T2 [4], which was recently finalized. Hybrid DVB-SH standard [5] has been developed in order to promise upgraded services to mobile and vehicle users via satellite link. The Geneva 2006 (GE-06) Agreement [6] regulates frequency usage in the broadcast bands of Europe, the digital transition to DVB and the analogue switch-off processes.

Transition from analogue TV to DVB is a complex feat. Frequency channels for new DTT programs must be allocated, safeguards must be taken to protect existing services from interference, equipment at transmission stations/towers must be changed, new digital services must be launched and viewers must be informed of the changes. The broadcast network must be planned, swapped and optimized. The role of network coverage and DVB quality measurements is absolutely important.

A crucial point of research is the quality of service (QoS) characterization of operating systems based on orthogonal frequency division multiplexing (OFDM) technology in a mobile radio environment. The objectives of our work are:
1. A short survey on audio-visual quality evaluation algorithms of the DVB user experience.

2. Key performance indicators (KPI's) that are critical for measurement like received RF signal power (RxLev), Carrier to Noise Ratio (CNR), Bit Error Rate (BER), Modulation Error Rate (MER), Frame Error Rate, Multi-Protocol Encapsulation (MPE) FER.

3. Experimental equipment for conducting indoor and outdoor measurements campaign.

4. QoS analysis procedures are proposed for DVB systems characterization.

Finally, we conclude with the importance of a field campaign in practical performance evaluation and optimization of a DVB radio network and possibly in benchmarking of DTT operators. The overall measurement methodology can be performed in the case of DVB-T2 or DVB-SH trial or in-deployment networks.

The rest of our research paper is organized as follows. The next section covers the major DVB systems for fixed (DVB-T/T2) and mobile/handheld (DVB-H/SH) reception. Section 3 gives an overview of key performance indicators and an experimental measurement campaign set-up and Section 4 provides QoS measurement campaign methodology. Finally, the conclusions and future work are drawn in Section 5.

\footnotetext{
* Charalampos N. Pitas, Athanasios D. Panagopoulos, Philip Constantinou

Mobile Radiocommunications Laboratory, Faculty of Electrical and Computer Engineering, National Technical University of Athens, Greece,

E-mail: chpitas@mobile.ntua.gr
} 


\section{DVB Systems}

\subsection{Terrestrial DVB Standards}

\subsubsection{DVB-T Standard}

In DVB-T standard, OFDM has been adopted in order to split the digital data stream into a large number of slower digital streams, each of which digitally modulate a set of closely spaced adjacent carrier frequencies. There are two choices for the number of carriers known as $2 \mathrm{~K}$-mode and $8 \mathrm{~K}$-mode with $1.7054 \mathrm{kHz}$ carriers and $6.8171 \mathrm{kHz}$ carriers respectively. Also, there are three available modulation schemes: QPSK, 16QAM, 64QAM.

DVB-T delivers data in a series of discrete blocks at the symbol rate. DVB-T is a COFDM transmission technique which includes the use of a Guard Interval. It allows the receiver to cope with strong multipath situations as it happens in case of mobile and vehicular environments. DVB-T also supports single-frequency network (SFN) operation, where transmitters carrying the same data operate on the same frequency in a geographical domain. Signals from each transmitter in the SFN need to be accurately time-aligned, which is done by sync information in the stream and timing at each transmitter referenced to GPS. The length of the Guard Interval can be chosen. It is a tradeoff between data rate and SFN capability. The longer the guard interval the larger is the potential SFN area without creating inter-symbol interference (ISI).

Specification [7] gives details of source coding methods for MPEG-2 and, more recently, H.264/MPEG-4 AVC [8] as well as audio encoding systems. Di Bari R. et al. in [9] conducted a measurement campaign for mobile DVB-T/H system.

\subsubsection{DVB-T2 Standard}

DVB-T2 [10] is the second generation of DVB-T and uses also OFDM modulation with a large number of sub-carriers delivering a robust signal. DVB-T2 uses LDPC (low density parity check) coding combined with $\mathrm{BCH}$ (Bose-Chaudhuri-Hocquengham) coding. The key DVB-T2 points are:

1. Rotated Constellations, which provide significant additional robustness in difficult radio channels.

2. Multiple Physical Layer Pipes that allow separate adjustment of the robustness of each delivered service within a channel to meet the required reception conditions (e.g. in-door or rooftop antenna).

3. Alamouti coding which is a transmitter diversity method improves coverage in small-scale SFNs.

4. Extended interleaving, including bit, cell, time and frequency interleaving.

5. Future Extension Frames allow the standard to be compatibly enhanced in the future.

As a result, DVB-T2 supports a much higher data rate than DVB-T as well as a much more robust signal. Eizmendi I. et al. in
[11] conducted HDTV field trials using DVB-T and DVB-T2 broadcasting systems.

\subsection{Handheld DVB Standards}

\subsubsection{DVB-H Standard}

DVB-H is a technical specification for the transmission of digital TV to handheld receivers such as mobile telephones and PDAs. It is designed to enable the efficient delivery of IP-encapsulated data over terrestrial networks. DVB-H is closely related to DVB-T and includes modifications dealing with data broadcasting, service information, mobile TV, video streaming in general and file downloads, all targeted at handheld receivers that would operate with a limited battery life and in difficult reception conditions. The most important of these were that there should be a significant power saving in the receiver compared to DVB-T, excellent performance and robustness in a cellular environment, and enhanced support for single antenna reception in single frequency networks (SFNs).

DVB-H [12] is an extension of DVB-T with some backwards compatibility. It uses a mechanism called multi-protocol encapsulation (MPE), making it possible to transport data network protocols on top of MPEG-2 transport streams. A forward error correction (FEC) scheme is used in conjunction with this to improve the robustness and thus mobility of the signal. In addition to the $2 \mathrm{~K}$ and $8 \mathrm{~K}$ modes available in DVB-T, a $4 \mathrm{k}$ mode is added to DVB-H giving increased flexibility for network design. Another essential element of DVB-H is Time Slicing, the main technique used to achieve the required power savings. Each individual TV service in a DVB-H signal is transmitted in bursts allowing the receiver to go into sleep mode, only waking up when the service to which it is "tuned" is transmitted. Statistical multiplexing is also possible in DVB-H, ensuring optimum use of bandwidth to deliver services. DVB-H is designed for use in Bands III, IV and V as well as L-band.

\subsubsection{DVB-SH Standard}

DVB-SH [13] is the name of a transmission system standard designed to deliver video, audio and data services to vehicles and handheld devices. The key feature of DVB-SH is that it is a hybrid satellite/terrestrial system that allows the use of a satellite to achieve coverage of large regions or even a whole country. In areas where direct reception of the satellite signal is not possible, terrestrial gap filler can be used seamlessly to provide coverage. It is designed to use frequencies below $3 \mathrm{GHz}$, typically around $2.2 \mathrm{GHz}$ [14].

The combination of a satellite and a terrestrial complement delivers wide coverage to mobile terminals which implement the TDM and OFDM modes of SH, a combination of SH and DVB$\mathrm{H}$, or simply the OFDM mode of DVB-SH operating in SFN. Key to deployment will be DVB-SH's interface with the existing DVBIPDC layer and the services based on it. Cohen M. et al. in [15] 
tested through various trials, including terrestrial only reception, satellite only reception and hybrid reception.

\section{Quality Measurements of DVB Radio Systems}

\subsection{Multimedia Quality of Experience}

\subsubsection{Audio Quality Evaluation}

Perceptual Evaluation of Audio Quality (PEAQ) algorithm was standardized by ITU-R for objectively measuring perceived audio quality. PEAQ simulate perceptual properties of the human ear and integrate multiple model output variables (MOV) into a single metric. PEAQ results principally model mean opinion scores (MOS).

Perceptual evaluation of speech quality (PESQ) algorithm supports only narrowband ( $4 \mathrm{kHz}$ bandwidth) speech signals while it does not support wideband applications used in contemporary multimedia communication systems.

Perceptual objective listening quality analysis (POLQA) is the new standardized algorithm for fixed, mobile and IP based networks POLQA has been selected to form the new ITU-T voice quality testing standard, P.863, and will be used with HD Voice, $3 \mathrm{G}$ and $4 \mathrm{G} / \mathrm{LTE}$

\subsubsection{Visual Quality Evaluation}

Objective video evaluation techniques are mathematical models that approximate results of subjective quality assessment, but are based on criteria and metrics that can be measured objectively and automatically evaluated by a computer program. Objective methods are classified based on the availability of the original video signal, which is considered to be of high quality (generally not compressed) Therefore, they can be classified as a) Full Reference methods (FR) b) Reduced Reference Models, and c) No Reference Models.

Recently, ITU-T standardized perceptual visual quality measurement techniques for multimedia services over digital cable television networks in the presence of a reduced bandwidth reference, J.246 [16], as well as objective perceptual multimedia video quality measurement in the presence of a full reference, J.247 [17].

A no-reference model has been proposed by ITU. Video quality $M O S_{\text {visual }}$ is computed using the video quality parameters:

$$
\operatorname{MOS}_{\text {visual }}=1+I_{\text {coding }} \times \exp \left(-\frac{P_{p l v}}{D_{P_{p l v}}}\right)
$$

where

- $I_{\text {coding }}$ represents the basic video quality affected by the coding distortion under a combination of video bit rate $\left(B r_{V}[\mathrm{kbit} / \mathrm{s}]\right)$ and video frame rate $\left(F r_{V}[\mathrm{fps}]\right)$.

- $P_{p / v}$ is the packet loss rate,

- Dplv is the packet loss robustness factor due to packet loss.

\subsubsection{Multimedia Quality Evaluation}

A subjective overall audiovisual quality, $M O S_{a v}$, assessment method for multimedia applications is a mapping across laboratories from the separate audio, $M O S_{a}$, and video, $M O S_{v}$, quality was found to be [18]:

$$
\operatorname{MOS}_{a v}=\left(c_{1}+M O S_{a}\right) \times\left(c_{2}+M O S_{v}\right)
$$

Total multimedia quality index, $M O S_{m m}$, can be computed by the audio-visual delay, $\mathrm{MOS}_{d}$, according to the following equations:

$$
\begin{aligned}
& \operatorname{MOS}_{d}=c_{3}+c_{4} \times \exp \left(c_{5} \times d / c_{6}\right) \\
& \operatorname{MOS}_{m m}=\left(c_{7}+\operatorname{MOS}_{a v}\right) \times\left(c_{8}+\operatorname{MOS}_{d}\right)
\end{aligned}
$$

where $c_{i} \forall \in[1,8]$ are constants, and $d$ is the "lip-sync" delay.

Recently, Singh KD and Rubino G. in [19] proposed no-reference QoE monitoring methods in DVB-H networks.

\subsection{Radio Quality Aspects}

Measurements guidelines for quality aspects in DVB-T systems are described in ETSI TR 101290 [20]. Analytically:

\subsubsection{System availability}

The system availability describes the long-term quality of the complete digital transmission system from MPEG-2 encoder to the measurement point.

\subsubsection{Link availability}

The link availability describes the long term quality of a specified link in a digital transmission chain. It could be used as a quality of service parameter in contracts between network operators and program providers.

\subsubsection{RF/IF signal power}

Level measurement is needed to set up a network. The signal power, or wanted power, is defined as the mean power of the selected signal as would be measured with a thermal power sensor. Care should be taken to limit the measurement to the bandwidth of the wanted signal. When using a spectrum analyzer or a calibrated receiver, it should integrate the signal power within the nominal bandwidth of the signal. 


\subsubsection{Noise power}

Noise is a significant impairment in a transmission network The noise power (mean power), or unwanted power, is measured with a spectrum analyzer (out of service) or an estimate is obtained from the IQ diagram (in service). The noise level is specified using either the occupied bandwidth of the signal, which is equal to the symbol rate.

\subsubsection{BER before RS decoder}

The Bit Error Rate (BER) is the primary parameter which describes the quality of the digital transmission link.

\subsubsection{Bit error count after RS}

BER measure whether the MPEG-2 TS is quasi error free.

The same principle as used for the "Out of service measurement" of the "BER before the Reed-Solomon decoder", with the modification that the result is presented as an error count rather than a ratio. The receiver only has to compare the received TS packets with the Null packets.

\subsubsection{I-Q signal analysis}

\section{Assuming:}

- a constellation diagram of $M$ symbol points; and

- a measurement sample of $N$ data points, where $\mathrm{N}$ is sufficiently larger than $\mathrm{M}$ to deliver the wanted measurement accuracy; and - the co-ordinates of each received data point $j$ being $I_{j}+\delta I_{j}$, $Q_{j}+\delta Q_{j}$ where $I$ and $Q$ are the co-ordinates of the ideal symbol point and $\delta I$ and $\delta Q$ are the offsets forming the error vector of the data point.

Modulation Error Ratio (MER) and the related Error Vector Magnitude (EVM) are calculated from all $N$ data points without special pre-calculation for the data belonging to the $M$ symbol points.

With the aim of separating individual influences from the received data, for each point $i$ of the $M$ symbol points the mean distance $d_{i}$ and the distribution $\sigma_{i}$ can be calculated from those $\delta I_{j}, \delta Q_{j}$ belonging to the point $i$.

From the $M$ values $\left\{d_{1}, d_{2}, \ldots, d_{M}\right\}$ the influences /parameters:

- origin offset;

- amplitude Imbalance (AI); and

- quadrature Error (QE),

can be extracted and removed from the di values, allowing to calculate the Residual Target Error (RTE) with the same algorithm as the System Target Error (STE) from $\left\{d_{1}, d_{2}, \ldots, d_{M}\right\}$.

From the statistical distribution of the $M$ clouds parameters:
- phase jitter; and

- CW interferer.

may be extracted. The remaining clouds (after elimination of the above two influences) are assumed to be due to Gaussian noise only and are the basis for calculation of the signal-to-noise ratio. The parameter may include - besides noise - also some other disturbing effects, like small non-coherent interferers or residual errors from the equalizer. From the SNR value the Carrier/Noise value can be estimated.

- origin offset;

- amplitude Imbalance (AI); and

- quadrature Error (QE),

can be extracted and removed from the di values, allowing to calculate the Residual Target Error (RTE) with the same algorithm as the System Target Error (STE) from $\left\{d_{1}, d_{2}, \ldots, d_{M}\right\}$.

From the statistical distribution of the $M$ clouds parameters:

- phase jitter; and

- CW interferer

may be extracted. The remaining clouds (after elimination of the above two influences) are assumed to be due to Gaussian noise only and are the basis for calculation of the signal-to-noise ratio. The parameter may include - besides noise - also some other disturbing effects, like small non-coherent interferers or residual errors from the equalizer. From the SNR value the Carrier/Noise value can be estimated.

$$
S N R=10 \times \log _{10}\left\{\frac{\frac{1}{N} \sum_{j=1}^{n}\left(I_{j}^{2}+Q_{j}^{2}\right)}{\frac{1}{N} \sum_{j=1}^{n}\left(\sigma I_{j}^{2}+\sigma Q_{j}^{2}\right)}\right\}
$$

Fig.1 depicts constellation diagrams for a double diversity receiver in case of excellent, good and bad coverage conditions.

- Modulation Error Ratio (MER)

MER provides a single "figure of merit" analysis of the received signal. This figure is computed to include the total signal degradation likely to be present at the input of a commercial receiver's decision circuits and so give an indication of the ability of that receiver to correctly decode the signal.

It should be reconsidered that MER is just one way of computing a "figure of merit" for a vector modulated signal. Another "figure of merit" calculation is Error Vector Magnitude (EVM). It is also shown that MER and EVM are closely related and that one can generally be computed from the other. MER is the preferred first choice for various reasons.

$$
M E R=10 \times \log _{10}\left\{\frac{\sum_{j=1}^{n}\left(I_{j}^{2}+Q_{j}^{2}\right)}{\sum_{j=1}^{n}\left(\delta I_{j}^{2}+\delta Q_{j}^{2}\right)}\right\}
$$




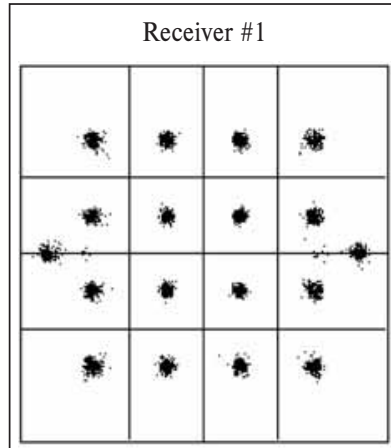

(a)

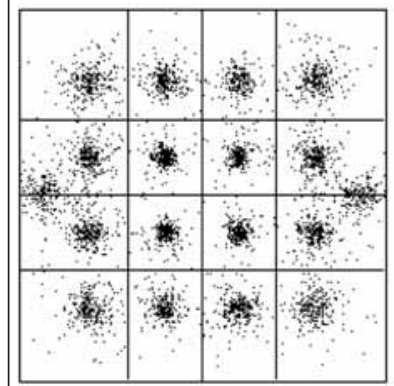

(c)

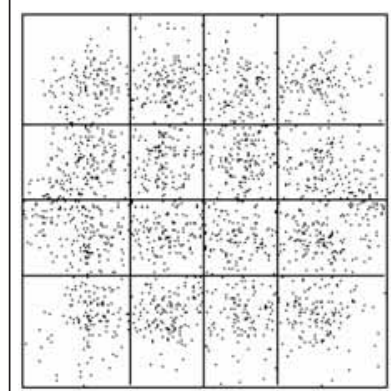

(e)

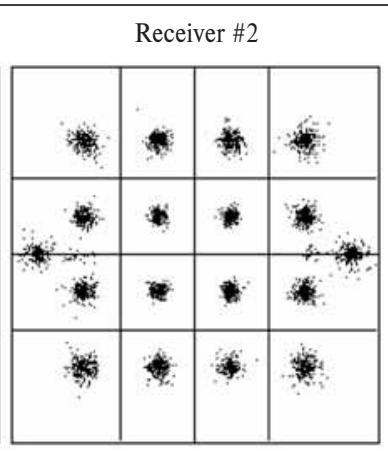

(b)

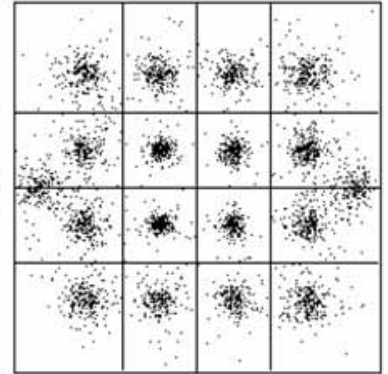

(d)

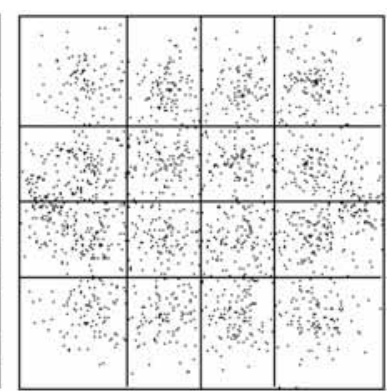

(f)
Fig. 1 Constellation diagram of the diversity $D V B-T / H$ receiver with two RF input ports in case of excellent (a-b), good (c-d) and bad coverage (e-f)

\section{- System Target Error (STE)}

The displacement of the centers of the clouds in a constellation diagram from their ideal symbol point reduces the noise immunity of the system and indicates the presence of special kind of distortions like Carrier Suppression, Amplitude Imbalance, Quadrature Error (QE). STE gives a global indication about the overall distortion present on the raw data received by the system.

From the magnitude of the $M$ Target Error Vectors calculate the mean value and the standard deviation (normalized to $S_{r m s}$, defined as the RMS amplitude value of the points in the constellation), obtaining the System Target Error Mean (STEM) and the System Target Error Deviation (STED) as follows:

$$
\begin{aligned}
& S_{r m s}=\sqrt{\frac{\sum_{j=1}^{N}\left(I_{j}^{2}+Q_{j}^{2}\right)}{N}} \\
& S T E M=\frac{\sum_{j=1}^{N}\left|\bar{d}_{j}\right|}{M \times S_{r m s}} \\
& S T E D=\sqrt{\frac{\sum_{j=1}^{n}\left|\bar{d}_{j}\right|^{2}}{M \times S_{r m s}^{2}}}-S T E M^{2}
\end{aligned}
$$

\section{- Carrier suppression}

A residual carrier is an unwanted coherent $\mathrm{CW}$ signal added to the QAM signal. It may have been produced by DC offset voltages of the modulating I and/or Q signal or by crosstalk from the modulating carrier within the modulator.

\section{- Residual Target Error (RTE)}

The RTE is a subset of the distortions measured as System Target Error (STE) with influences of Carrier Suppression, Amplitude Imbalance, and Quadrature Error (QE) removed. The remaining distortions may result mainly from non-linear distortions.

\section{- Coherent interferer}

Coherent interferers are usually measured with a spectrum analyzer. From the statistical distribution of the clouds, the amplitude of the interferer can be calculated if it is above a certain limit.

\section{Measurements System and Campaign}

\subsection{Measurements System}

The functional block diagram of $R \& S T S M-D V B$ [21] (see Fig. 2) highlights two receiver channels in the form of any of the following:

- A diversity receiver with two different antennas connected to the two RF inputs that are $50 \Omega$ unbalanced.

- Two high-performance tuners that are compatible with VHF (Ch 5 - 12) and UHF (Ch 21 - 69). Channel bandwidth could be $5 \mathrm{MHz}, 6 \mathrm{MHz}, 7 \mathrm{MHz}$ or $8 \mathrm{MHz}$. The RF input is $50 \Omega$ unbalanced.

- Two discrete DVB-T demodulators suitable for mobile, portable and stationary applications.

- Two asynchronous serial interfaces (ASI).

- A configurable complex programmable logic device (CPLD) which is used for MPEG stream switching.

The measurements system was installed in a vehicle as it is shown in Fig. 3. The two antennas were racked on the roof and the RF cables were carefully driven through a window. A netbook 


\begin{tabular}{|c|c|}
\multicolumn{1}{c}{} & \multicolumn{1}{c}{ Antenna } \\
$Y_{\# 1}$ & $\begin{array}{c}\text { Antenna } \\
Y_{\# 2}\end{array}$ \\
\hline RF IN \#1 & RF IN \#2 \\
\hline Tuner \#1 & Tuner \#2 \\
\hline DVB-T/H & DVB-T/H \\
Demodulator & $\begin{array}{c}\text { Demodulator } \\
\# 2\end{array}$ \\
$\# 1$ & Redundancy and ASI \\
Management CPLD \\
\hline ASI IF & ASI IF \\
$\# 1$ & OUT \#2 \\
\hline OUT \#1 & OU
\end{tabular}

Fig. 2 Functional block diagram of the DVB-T/H diversity test system for drive-test measurement campaign

was used for measurements system control and measurements data storage. The power supply of the netbook and the drive-test equipment was directly from the vehicle's battery. All measurements were triggered with an external GPS receiver.

\subsection{Measurements Campaign}

- Measurements Data Handling. A set of processes shall be defined for measurements data handling. During a measurement campaign, large sets of data are going to be produced in a daily basis. The measurement team shall organize the produced measurements and deliver them to the analysis team to take into account security and integrity issues. No measurement data shall be lost or damaged. Data handling must be reliable end-to-end. Back-up process shall be defined by the analysis team.

- Verification Tests. A set of verification tests shall be executed before measurement campaign. A crucial point is the performance of antennas as well as the feeding RF cables. These components have to be in-lab tested via a network analyzer in order to characterize indicators like return or reflection loss $(R L)$ of signal power from the reflection caused at a discontinuity in the RF cable, the reflection coefficient $(I)$, or alternatively the standing wave ratio $(S W R)$ by using a SWR meter.

- Route Planning. A route plan shall be defined before a measurement campaign starts. A measurement campaign is usually a longtime drive-test where urban, suburban and rural routes as well as major motorways, islands and touristic areas are to be measured. It is impossible to measure all around the territory. So, a representative subset of routes shall be chosen under population and geographic criteria. A time schedule shall be agreed according to the route plan. Besides, hotspots like shopping centers, airports, ports, railway stations and central town squares should be included to the schedule for static measurements.

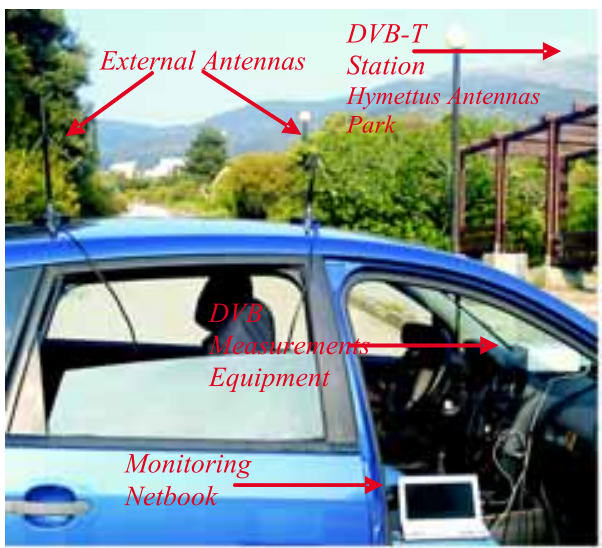

Fig. 3 Details of the measurements equipment installation before drive-test campaign
- Technical reports delivery. Technical reporting is a crucial process during a measurement campaign of DVB radio networks. Analysis may be performed by type of route (town, motorway, touristic area, or island) as well as by wide geographic area (prefecture, region, allotment). A final executive/summary report shall be delivered at the end of the campaign. It is important to select representative and comparable key performance indicators (KPIs) for technical reporting. Post processing analysis of the KPIs is usually based on statistics. Results presentation is usually performed using coverage maps, probability and cumulative density function (PDF/ CDF) diagrams and pivot tables as well.

\section{Conclusions}

The scope of our paper was to propose a methodology for QoS characterization of real world digital video broadcasting (DVB) networks taking into account audio-visual quality. DVB standards for fixed terrestrial and mobile handheld transmission have been already commercially adopted for multimedia broadcasting deployment. Offspring DVB-T2 and DVB-SH technologies will be adopted by operators for broadband multimedia services. Analytically, we presented critical QoS indicators both for radio coverage and performance assessment. An experiment setup for DVB measurement campaign was described based on special laboratory equipment with diversity reception capability. Measurements analysis procedures were proposed for performance evaluation and of radio broadcasting networks.

\section{Acknowledgements}

The experimental equipment was acquired during the "05AKM $\Omega$ N95" Project; financially supported by the General Secretariat of Research and Technology, Greek Ministry of Development (funded by E.U. and National financial resources). 


\section{References}

[1] Digital Video Broadcasting Project (DVB). http://www.dvb.org/.

[2] ETSI EN 101 190: Digital Video Broadcasting (DVB); Implementation Guidelines for DVB Terrestrial Services; Transmission Aspects (DVB-T). V.1.3.1, Oct. 2008.

[3] ETSI TR 102 377: Digital Video Broadcasting (DVB); Implementation Guidelines for DVB Handheld Services (DVB-H). V1.4.1, Jun. 2009.

[4] ETSI TR 102 831: Digital Video Broadcasting (DVB); Implementation Guidelines for a Second Generation Digital Terrestrial Television Broadcasting System (DVB-T2). V0.10.4 (DRAFT), Jun. 2010.

[5] ETSI TS 102 584: Digital Video Broadcasting (DVB); DVB-SH Implementation Guidelines. V1.2.1, Jan. 2011.

[6] ITU-R: Final Acts of the Regional Radiocomm Conference for Planning of the Digital Terrestrial Broadcasting Service in Parts of Regions 1 and 3, in the Frequency Bands 174-230 MHz and 470-862 MHz. RRC-06, 2006.

[7] ETSI EN 300 744: Digital Video Broadcasting (DVB); Framing Structure, Channel Coding and Modulation for Digital Terrestrial Television (DVB-T). V1.6.1, Jan. 2009.

[8] ITU-T H.264: Advanced Video Coding for Generic Audiovisual Services. 2008.

[9] DI BARI, R. et al: Measurement Campaign on Transmit Delay Diversity for Mobile DVB-T/H Systems”, IEEE Trans. on Broadcasting, 2010, Vol. 56 (3), pp. 369-378.

[10] ETSI EN 302 755: Digital Video Broadcasting (DVB); Frame Structure Channel Coding and Modulation for a Second Generation Digital Terrestrial Television Broadcasting System (DVB-T2). V1.2.1, Oct. 2010.

[11] EIZMENDI, I. et al.: HDTV Field Trials Using DVB-T and DVB-T2 Broadcasting Systems. In Proc. IEEE Int '1 Symp. on Broadband Multimedia Systems and Broadcasting (BMSB'10), 2010, pp. 1-5

[12] ETSI EN 302 304: Digital Video Broadcasting (DVB); Transmission System for Handheld Terminals (DVB-H). V1.1.1, Nov. 2004.

[13] ETSI EN 302 583: Digital Video Broadcasting (DVB); System Specifications for Satellite services to Handheld Devices (SH) Below 3 $G H z(D V B-S H)$. V1.1.0, Jan. 2008.

[14] ETSI TS 102 584: Digital Video Broadcasting (DVB); DVB-SH Implementation Guidelines. V1.2.1, Jan. 2011.

[15] COHEN, M. et al.: DVB-SH Field Trials Measurements Results. In Proc. $11^{\text {th }}$ SPSC, 2010, pp. 530-537.

[16] ITU-T J.246: Perceptual Audiovisual Quality Measurement Techniques for Multimedia Services over Digital Cable Television Networks in the Presence of a Reduced Bandwidth Reference. 2008.

[17] ITU-T J.247: Objective Perceptual Multimedia Video Quality Measurement in the Presence of a Full Reference. Apr. 2007.

[18] ITU-T P.911: Audiovisual Quality in Multimedia Services; Subjective Audiovisual Quality Assessment Methods for Multimedia Applications, Dec. 1998.

[19] SINGH, K.D., RUBINO G.: No-reference Quality of Experience Monitoring in DVB-H Networks. In Proc. of Wirel Telecommun Symp (WTS'10), 2010, pp.1-6.

[20] ETSI TR 101 290: Digital Video Broadcasting (DVB); Measurement Guidelines for DVB Systems. V.1.2.1, May 2001.

[21] ROHDE \& SCHWARZ: R\&S TSM-DVB DVB-T/H Diversity Test Receiver. http://www2.rohde-schwarz.com/. 